\title{
Editorial
}

\section{Wireless Location Technologies and Applications}

\author{
Thomas Kaiser, ${ }^{1}$ Ian Oppermann, ${ }^{2}$ and Domenico Porcino ${ }^{3}$ \\ ${ }^{1}$ Department of Electrical Engineering and Information Technology, Communication Systems Group, \\ Leibniz University of Hannover, Appelstraße 9A, 30167 Hannover, Germany \\ ${ }^{2}$ Centre for Wireless Communications (CWC), University of Oulu, Oulu 90014, Finland \\ ${ }^{3}$ Wireless Group, Philips Research Laboratories, Cross Oak Lane, Redhill, Surrey RH1 5HA, UK
}

Received 30 July 2006; Accepted 30 July 2006

Copyright (C) 2006 Thomas Kaiser et al. This is an open access article distributed under the Creative Commons Attribution License, which permits unrestricted use, distribution, and reproduction in any medium, provided the original work is properly cited.

Technologies for location and tracking are fast maturing with an array of new techniques, new algorithms, system designs, and trials bringing location-based services into everyday life. This once niche technology is maturing and gaining wider acceptance, thus finding more and more applications every day.

The development of communications systems that include location and tracking capabilities has generated great interest also in the fields of cellular and wireless local/personal area networks. A host of potential new services can be enabled by suitably accurate location and tracking facilities in conjunction with appropriate communications and data transfer platforms. Researchers worldwide are pushing the boundaries of precision, low-cost, component reusability, and ease of field deployment.

From established radio techniques, such as WLAN (e.g., IEEE $802.11 \mathrm{a} / \mathrm{b} / \mathrm{g}$ ) to emerging WPAN networks (e.g., Bluetooth and IEEE 802.15.4) to newer ultra-wideband (UWB) systems (e.g., IEEE 802.15.3a and IEEE 802.15.4a), and further previously unconsidered technologies such as digital TV signals, a common denominator to drive adoption and growth is implementing innovative services on top of data transfer. Whether the positioning techniques are based on signal strength, time of flight, or fingerprinting techniques, they offer the potential to improve and optimize traditional services (as diverse as flight navigation or data routing) or bring to life new applications relying on the knowledge of the location of the wireless nodes.

A large number of issues must be addressed to move from coarse delay measurement to useful range estimation for tracking purposes. Issues include generation of accurate delay/ranging estimates, proper operation in dense multipath environments, delay/ranging information sharing between nodes, computationally efficient algorithms, algorithms for low infrastructure environments, dealing with nonline of sight as well as integration with usable applications. The aim of this special issue is to address the state of the art in wireless location technologies and applications with particular emphasis on accurate results in low infrastructure environments. We classified the 16 contributions into 3 major categories, namely, performance analysis, algorithms, and systems/ applications.

"Systematic errors and location accuracy in wireless networks" by Harri Saarnisaari and Timo Bräysy is the first paper in the category "performance analysis" and provides a unified error-propagation-law-based tool to analyze measurement and systematic errors. The tool is used in order to compare hyperbolic (time delay based), direction finding, received signal strength (RSS), and relative RSS (RRSS), location systems. The obtained analytical results verify several intuitive expectations, for example, that the hyperbolic methods are sensitive to network synchronization errors.

In the paper "Autonomous positioning techniques based on Cramér-Rao lower bound analysis" by Mats Rydström et al. the problem of autonomously locating asynchronous sensor nodes in a wireless network is addressed, where the number of processing resources needed will be minimized. An optimal scheme for sensor clock-offset cancellation is proposed in the first part of the paper, while the second part concerns about a divide-and-conquer approach for suboptimal positioning, which is also based on the corresponding CramérRao lower bound (CRB).

The next paper "Cramér-Rao type bounds for localization" by Cheng Chang and Anant Sahai studies the localization problem in sensor networks, which is also based on the fundamental CRB for noisy range measurements. They investigate the impact of a priori knowledge of the postion of a few reference nodes and prove some invariances of the CRB 
as well as its extension to large networks for different wireless signal propagation models.

The last paper of this category is "Performance evaluation of indoor localization techniques based on RF power measurements from active or passive devices" by Damiano De Luca et al. In this contribution the authors discussed the performance of networks of indoor RF identification (RFID) localization units based on signal strength analysis on a fixed grid against prerecorded RF maps. The paper discusses also the maintenance and installation costs of active versus passive tags and the effects of the tag population.

When considering tracking of objects, many position calculations must be performed per second representing a considerable computational burden. Techniques which reduce complexity of a single positioning calculation, or which make use of the availability of other information to reduce the need for the number of calculations per second can yield considerable savings. This is particularly important for handheld or battery powered devices. These issues are addressed in the next category "algorithms."

The paper "A constrained least squares approach to mobile positioning: algorithms and optimality" by K. W. Cheung et al. addresses a complexity constrained approach to position calculation which supports relatively high accuracy.

Many location-based techniques are dependent on determining the angle or time of arrival of multiple signals. The accuracy of identifying the arrival time or angle directly impacts the accuracy of the positioning results. The paper "Adaptive subchip multipath resolving for wireless location systems" by Nabil R. Yousef et al. describes a technique for improving the accuracy of identification of multipath time/angle of arrival which may then be used to significantly improve the position estimate.

Similarly, the paper "Time of arrival estimation for UWB localizers in realistic environments" by Chiara Falsi et al. examines the time of arrival estimation problem (almost trivial under ideal conditions) under the real world constraints of imperfect reception, noise, and other mitigating factors.

In "Robust estimator for non-line-of-sight error mitigation in indoor localization" by R. Casas et al. a robust regression techniques to detect and reject nonline-of-sight (NLoS) measures is proposed. The technique is suitable in environments with little infrastructure requirements and relies on multilateration and a least-median-of-squares criterion.

In the paper "Application of beamforming in wireless location estimation" by Kamran Sayrafian-Pour and Dominik Kaspar it is assumed that the receiver is equipped with a circular antenna array so that beamforming becomes feasible. This property basically enables a single receiver to estimate and track the position of a mobile transmitter. The feasibility of this methodology is investigated and the accuracy improvements are demonstrated.

A similar approach is considered in the paper "Blind mobile positioning in urban environment based on ray-tracing analysis" by Shohei Kikuchi et al. The signals received by a single base station equipped with an antenna array are combined with an a priori ray-tracing analysis that is based on highly accurate three-dimensional terrain data. Moreover, the approach is entirely "blind," so that neither a training sequence nor a specific waveform from the mobile terminal is required.

The paper "Particle filtering algorithms for tracking a maneuvering target using a network of wireless dynamic sensors" by Joaquín Míguez and Antonio Artés-Rodríguez takes a novel approach and explores the application of a complex yet reasonably accurate technique to wireless network.

The paper "Feedforward delay estimators in adverse multipath propagation for Galileo and modernized GPS signals" by Elena Simona Lohan et al. is a useful reference for the researchers engaged in the global navigation satellite systems (GNSS). It presents a good analysis (and attempt of reduction) of the severe multipath effects on the line of sight delay measurement via the use of feedforward delay estimation techniques. The authors present an extensive review of known methods and extend them to BOC-modulated pseudorandom codes, also taking into account the needed computational resources in state-of-the-art, low-power DSPs.

The third category of papers in this special issue touches on "systems and applications" involving location-based systems.

The paper "A new position location system using DTV transmitter identification watermark signals" by Xianbin Wang et al. presents the innovative idea of using transmitter identification RF watermarking by analyzing the DTV signals. These signals are transmitted at much higher power than satellite signals and can also be recorded with good accuracy indoors and could be used as a reference for timing and time of flight information. The performance of a test system has been tested via simulations and an initial field trial.

The paper "Practical network-based techniques for mobile positioning in UMTS" by Jakub Borkowski and Jukka Lempiäinen shows results of research on cellular positioning for $3 \mathrm{G}$ systems. Two main methods are presented and evaluated: the ECID + RTT (enhanced cell identification + round trip time) and the PCM (pilot correlation method). In both cases the authors show the merits (precision, system simplicity) and drawbacks (complexity costs, effects on system capacity) of each method showing how they could evolve in hybrid satellite-cellular positioning networks.

The paper "A new location estimation system for wireless networks based on linear discriminant functions and hidden Markov models" by Galo Nuño-Barrau and José M. PáezBorrallo examines a technique for applying structural information to enhance the performance of position estimation leading to improved performance in different network types. The work is applicable to a number of different technologies.

In the paper "An innovative gateway for indoor positioning” by G. F. Marias et al. a novel entity called gateway WLAN location center (GWLC) is proposed, which incorporates a unified framework for retrieving location data of users and objects in heterogeneous indoor and WLAN-based communication systems. The design principles and the functionality of the GWLC are elaborated and real experiments demonstrate the performance of the gateway.

The paper "Advanced integration of WiFi and inertial navigation systems for indoor mobile positioning" by 
Frédéric Evennou and François Marx presents an aided dead reckoning navigation structure and signal processing algorithms for self localization of an autonomous mobile device by fusing pedestrian deadreckoning and WiFi signal strength measurements. The benefits of the proposed architecture are evaluated and compared with the pure WiFi and inertial navigation systems.

Hundreds of researchers worldwide have contributed to this special issue submitting their latest research, reviewing papers, suggesting changes, and improvements in successive iterations. We would like to thank them all for a great effort over a year's work and collaboration. We would also like to send our special thanks to the publisher and the editor-inchief for their patience and useful suggestions along the path to the finalization of the issue.

We are confident that this special issue will become a useful reference for the current status of play and stimulate further research on wireless location technologies in the months to come. We wish all the best to all those following and working in this exciting positioning field!

Thomas Kaiser Ian Oppermann Domenico Porcino

Thomas Kaiser received the Ph.D. degree in 1995 with distinction and the German Habilitation degree in 2000, both from Gerhard Mercator University Duisburg and in electrical engineering. In the summer of 2005 he joined Stanford's Smart Antenna Research Group (SARG) as a Visiting Professor. Now he holds a Chair on communication systems at the University of Hannover, Germany, and is the Founder of the spin-off company mimoOn GmbH. He has published more than 100 papers and has coedited four books on ultra-wideband and smart antenna systems. He is the founding Editor-in-Chief of the IEEE Signal Processing Society E-Letter. His research interest focuses on applied signal processing with emphasis on multiantenna systems, especially its applicability to ultra-wideband systems.

Ian Oppermann is currently the Director of Performance Products within the Software Business Group of Nokia Networks, Finland. Within his product development portfolio are leading edge network planning and optimization tools for $2 \mathrm{G}$ and $3 \mathrm{G}$ technologies. Before joining Nokia, he was the Director of the Centre for Wireless Communications (CWC), a self-funded research centre in Oulu, Finland. At the CWC, he was

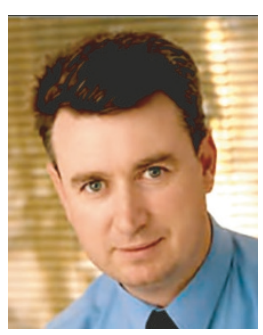
responsible for looking at "beyond 3G" mobile systems including ultra-wideband (UWB), cellular and noncellular networks. From 1996 to 2002, he was CTO of an Australian software development company developing network planning and optimization tools. $\mathrm{He}$ holds undergraduate degrees in science (1990) and electrical engineering (1992) from the University of Sydney, Australia. He also earned his Ph.D. degree from the University of Sydney in 1997, where his thesis explored CDMA physical layer technologies. In 2005, he completed an MBA at the University of London.
Domenico Porcino received his Dott. Ing. degree in electronics engineering from Politecnico di Torino, Italy, with main emphasis on mobile communications. He started his career with Philips Research Laboratories (PRL), UK, engaging in research and development activities for low-power radio devices. While at Philips, he has been involved in the design and testing of a transceiver chip for low-power wireless

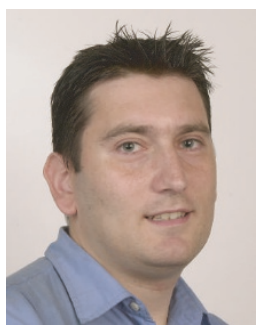
telemetry devices, simulation of novel RF receiver architectures and baseband processing for GPS receivers, and baseband development for third-generation mobile phones. For the last five years he has been leading PRL activities in the ultra-wideband (UWB) domain, looking at a variety of topics such as propagation, wideband antennas, regulations, ranging, and video streaming. Author of several publications and patent applications, he has also held coordinating roles in the ULTRAWAVES (EC IST FP5) and PULSERS (EC IST FP6) projects. 